\title{
Isothermal viscoelasticity and energy
}

\author{
Mária Minárová* \\ Faculty of Civil Engineering, Slovak University of Technology, 81005 Bratislava, Slovakia
}

\begin{abstract}
Within the scope of the linear viscoelasticity theory, the change in the inner energy of a viscoelastic body is induced either by heat exchange or by a work performance. The first law of thermodynamics, balance equation of a closed system is mostly referred when the thermodynamic consistency of some rheological model is required. Accordingly, within the frame of the isothermal viscoelastic investigation we just distinguish between the stored and dissipated energy. And this is the issue that the paper is focused on. Subjected to a load, the one degree of freedom viscoelastic models' behaviour is traced, together with the observation of the energy total, stored and dissipated. Nevertheless, the only stored energy in viscoelastic model is potential energy. General considerations are applied on Maxwell model subjected to the standard both creep and relaxation tests.
\end{abstract}

\section{Introduction}

This work is a continuation of author's investigation in the field, namely [1-3]. It is focused to the thermodynamic aspects of viscoelastic (VE) models. The exploration is carried out within the framework of the linear viscoelasticity theory. Viscoelastic bodies, represented by corresponding models are studied and the energy flow while the VE bodies are loaded. The isothermal state of a system is supposed.

\section{Viscoelastic models and related equations}

Viscoelasticity theory is a part of rheology, material science studying material which are neither purely elastic solids nor purely viscous liquids. Linear viscoelasticity fruitfully exploits the Boltzmann superposition principle.

\subsection{Fundamental viscoelastic elements}

Each viscoelastic model involves two types of fundamental elements, Hookean elastic matter $(\mathrm{H})$ characterised by linear dependence between stress and strain

\footnotetext{
* Corresponding author: maria.minarova@stuba.sk
} 


$$
\sigma(t)=E \varepsilon(t)
$$

and Newton viscous fluid $(\mathrm{N})$ characterised by linear dependence between stress and strain rate, see Fig.1.

$$
\sigma(t)=\eta \dot{\varepsilon}(t)
$$

with $E[P a]$ being elastic Young modulus and $\eta[P a . s]$ Newton viscous coefficient.

(H)

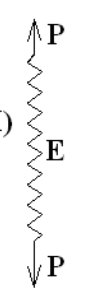

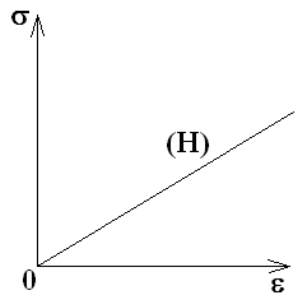
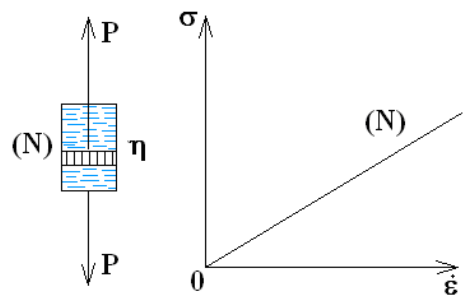

Fig. 1. Hookean $(\mathrm{H})$ and Newtonian $(\mathrm{N})$ matters - fundamental matters of a viscoelastic model [1].

Elastic and viscous elements within a VE model are connected in parallel or serially, as it is performed in Fig. 2. By using abbreviations (H) for Hookean elastic matter and $(\mathrm{N})$ for Newtonian viscous matter, together with signs | and - for parallel and serial connection respectively, we can write down the structural forms of arbitrary VE model. The simplest two element VE models are called Maxwell model $(\mathrm{M})=(\mathrm{H})-(\mathrm{N})$ and Kelvin-Voigt model $(\mathrm{KV})=(\mathrm{H}) \mid(\mathrm{N})$.
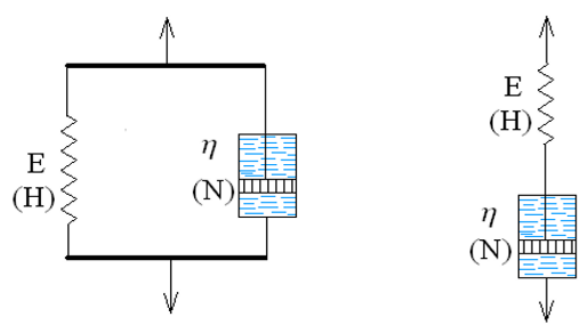

Fig. 2. Parallel (left) and serial (right) connection of fundamental matters yielding Kelvin-Voigt $(\mathrm{KV})=(\mathrm{H}) \mid(\mathrm{N})$ model and Maxwell model $(\mathrm{M})=(\mathrm{H})-(\mathrm{N})$, respectively.

In parallel connection, Fig. 2 left, total deformation is the same as the deformations of both involved elements and total stress is equal to the sum of particular values of stress.

$$
\begin{aligned}
& \varepsilon(t)=\varepsilon_{\mathrm{H}}(t)=\varepsilon_{\mathrm{N}}(t) \\
& \sigma(t)=\sigma_{\mathrm{H}}(t)+\sigma_{\mathrm{N}}(t)
\end{aligned}
$$

By coupling (3), (4) with (1) and (2) and by eliminating all indexed variables, we get the stress - strain relation of the model, an implicit form of constitutive equation of Kelvin-Voigt model:

$$
(K V): \sigma(t)=E \varepsilon(t)+\eta \dot{\varepsilon}(t)
$$


In serial connection, Fig. 2 right, total deformation is equal to the sum of both involved elements' deformations and total stress is of the same magnitude as the particular values of stresses of both fundamental elements involved. [4]

$$
\begin{aligned}
& \varepsilon(t)=\varepsilon_{\mathrm{H}}(t)+\varepsilon_{\mathrm{N}}(t) \\
& \sigma(t)=\sigma_{\mathrm{H}}(t)=\sigma_{\mathrm{N}}(t)
\end{aligned}
$$

When we couple (6), (7) with (1) and (2) and we eliminate all indexed variables, we get the stress - strain relation of the model, an implicit form of constitutive equation of Maxwell model:

$$
(M): \dot{\varepsilon}(t)=\frac{\dot{\sigma}(t)}{E}+\frac{\sigma(t)}{\eta}
$$

Both constitutive equations (5) and (8) are linear differential equations easily solvable with respect to either $\varepsilon$ or $\sigma$.

\section{Thermodynamics in rheology}

Each rheological element, accordingly a VE model, governed by a constitutive relation endowed by a potential energy $U_{\mathrm{p}} \geq 0$, has to be thermodynamically consistent. Hence the dissipation rate of the model energy has to be nonnegative [5]

$$
\dot{U}_{\mathrm{d}}(t)=\dot{U}(t)-\dot{U}_{\mathrm{p}}(t) \geq 0
$$

with $U$ being the total work of deformation, $U_{\mathrm{d}}$ the dissipated energy and $U_{\mathrm{p}}$ the potential (stored) energy.

\subsection{Stored and dissipated energy in VE models}

The total work of deformation in VE models is split to stored and dissipated energy. Generally, the stored energy can be potential and kinetic, nevertheless during viscoelastic investigation we consider the types of load causing negligible inertial impact. So further, alongside the text, we identify the stored energy with potential one. It is important to recall that all potential energy is stored due to elastic element involved; and all energy spent to viscous element deformation is dissipated, changed to heat. Indeed, as we have isothermal state, all dissipated energy is lost energy as far as any other movement on VE model concerns.

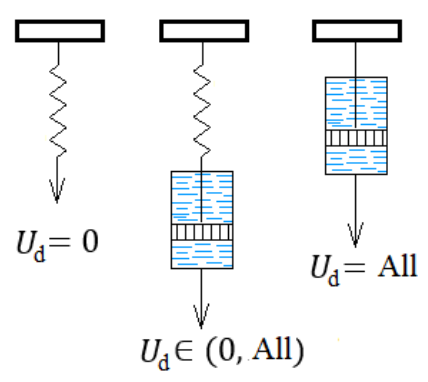

Fig. 3. Storing and dissipating of the energy [2]. 
Now we trace the total deformation energy flow during a load process and its partition to the potential and dissipated component having in mind the constitutive equation of VE model which expresses the dependence of the reaction on the action. First we are interested in a total work of deformation. The stress power of VE model, i.e. the rate of deformation work which is performed per time and volume unit, is [8]

$$
\dot{U}(t)=\sigma(t) \dot{\varepsilon}(t)
$$

Consequently, the deformation work is

$$
U(t)=U(0)+\int_{0}^{t} \dot{U}(a) \mathrm{d} a=U(0)+\int_{0}^{t} \sigma(a) \dot{\varepsilon}(a) \mathrm{d} a
$$

For the sake of expressing (9) and (10) in sense of $\varepsilon(t)$ or $\sigma(t)$, we can exploit so called time dependent material characteristics relaxation modulus $\tilde{E}(t)=\frac{\sigma(t)}{\varepsilon(t)}$ and compliance modulus $\tilde{C}(t)=\frac{\varepsilon(t)}{\sigma(t)}$ which are specific for specific load type for particular VE model. Then we can rewrite the total deformation energy rate and the total deformation energy itself in the form

$$
\begin{gathered}
\dot{U}(t)=\tilde{E}(t) \varepsilon(t) \dot{\varepsilon}(t)=\sigma(t) \frac{\mathrm{d}}{\mathrm{d} t}(\tilde{C}(t) \sigma(t)) \\
U(t)=U(0)+\int_{0}^{t} \tilde{E}(a) \varepsilon(a) \dot{\varepsilon}(a) \mathrm{d} a=U(0)+\int_{0}^{t} \sigma(a) \frac{\mathrm{d}}{\mathrm{d} t}(\tilde{C}(a) \sigma(a)) \mathrm{d} a
\end{gathered}
$$

Remark: General rules for the derivation of time dependent material characteristics are elaborated in [3] and synoptically provided in [2].

The dissipation energy rate and the dissipation energy itself of a VE model can be written as:

$$
\begin{gathered}
\dot{U}_{\mathrm{d}}(t)=\sigma_{\mathrm{N}}(t) \dot{\varepsilon}_{\mathrm{N}}(t)=\eta \dot{\varepsilon}_{\mathrm{N}}^{2}(t)=\eta^{-1}{\sigma_{\mathrm{N}}}^{2}(t) \\
U_{\mathrm{d}}(t)=U_{\mathrm{d}}(0)+\int_{0}^{t} \dot{U}_{\mathrm{d}}(a) \mathrm{d} a=U_{\mathrm{d}}(0)+\int_{0}^{t} \eta \dot{\varepsilon}_{\mathrm{N}}^{2}(a) \mathrm{d} a=U_{\mathrm{d}}(0)+\int_{0}^{t} \eta^{-1}{\sigma_{\mathrm{N}}}^{2}(a) \mathrm{d} a
\end{gathered}
$$

Thereafter, the potential (stored) energy rate and the potential energy itself is given as follows:

$$
\begin{gathered}
\dot{U}_{\mathrm{p}}(t)=\sigma_{\mathrm{H}}(t) \dot{\varepsilon}_{\mathrm{H}}(t)=E \varepsilon_{\mathrm{H}}(t) \dot{\varepsilon}_{\mathrm{H}}(t)=E^{-1} \sigma_{\mathrm{H}}(t) \dot{\sigma}_{\mathrm{H}}(t) \\
U_{\mathrm{p}}(t)=U_{\mathrm{p}}(0)+\int_{0}^{t} E \varepsilon_{\mathrm{H}}(a) \dot{\varepsilon}_{\mathrm{H}}(a) \mathrm{d} a=U_{\mathrm{p}}(0)+\int_{0}^{t} E^{-1} \sigma_{\mathrm{H}}(a) \dot{\sigma}_{\mathrm{H}}(a) \mathrm{d} a
\end{gathered}
$$

If there are more elastic or more viscous elements involved in the VE model, then the corresponding energies are summed up adequately.

Remark: Since the dashpot prevents the immediate deformation, a nonzero immediate initial deformation appears only in the case when an elastic element $(\mathrm{H})$ is connected serially in the VE model configuration. In such a case the initial dissipation energy is zero. Then $(\mathrm{H})$ stores 
all initial immediate potential energy $U_{\mathrm{p}}(0)=\frac{1}{2} E \varepsilon_{\mathrm{H}}{ }^{2}(0)=\frac{1}{2 E}{\sigma_{\mathrm{H}}}^{2}(0)$. Consequently, the potential energy is, [7]

$$
U_{\mathrm{p}}(t)=\frac{1}{2} E \varepsilon_{\mathrm{H}}^{2}(0)+\int_{0}^{t} E \varepsilon_{\mathrm{H}}(a) \dot{\varepsilon}_{\mathrm{H}}(a) \mathrm{d} a=\frac{1}{2 E}{\sigma_{\mathrm{H}}}^{2}(0)+\int_{0}^{t} E^{-1} \sigma_{\mathrm{H}}(a) \dot{\sigma}_{\mathrm{H}}(a) \mathrm{d} a
$$

Both integrals in (15) can be regarded (and evaluated) as Stjelties integrals as well:

$$
\begin{gathered}
\int_{0}^{t} E \varepsilon(a) \dot{\varepsilon}_{\mathrm{H}}(a) \mathrm{d} a=E \int_{\varepsilon(0)}^{\varepsilon(t)} \varepsilon_{\mathrm{H}}(a) \mathrm{d} \varepsilon(a)=\frac{1}{2} E \varepsilon_{\mathrm{H}}^{2}(t)-\frac{1}{2} E \varepsilon_{\mathrm{H}}^{2}(0) \\
\int_{0}^{t} E^{-1} \sigma_{\mathrm{H}}(a) \dot{\sigma}_{\mathrm{H}}(a) \mathrm{d} a=E^{-1} \int_{\sigma_{\mathrm{H}}(0)}^{\sigma_{\mathrm{H}}(t)} \sigma_{\mathrm{H}}(a) \mathrm{d} \sigma_{\mathrm{H}}(a)=\frac{1}{2 E}{\sigma_{\mathrm{H}}}^{2}(t)-\frac{1}{2 E} \sigma_{\mathrm{H}}{ }^{2}(0)
\end{gathered}
$$

\section{Energy of Maxwell model subjected to a load}

There are two typical lab tests - creep test and relaxation test obviously executed in material science in order to mutual comparison of rheological material. That is why we deal with the load typical for these tests.

Following consideration is focused on the Maxwell model exclusively. We study the energy quantification of the model tied up with the mechanical response of the model subjected to both standard loading tests. Herein we use the thermodynamic consideration provided in Chapter 3. As mentioned above, Maxwell model $(\mathrm{M})=(\mathrm{H})-(\mathrm{N})$ performed in Fig. 2 right, is governed by its constitutive relation (8).

\subsection{Creep test on Maxwell model - constant stress imposed}

When a constant stress is applied at (M) and maintained in time: $\sigma(t)=\sigma^{*} ; t \in\left(t_{0}, t_{1}\right)$, the responding deformation function is yielded as a solution of the simple differential equation

$$
\frac{\dot{\sigma}^{*}}{E}+\frac{\sigma^{*}}{\eta}=\dot{\varepsilon}(t) \text { in }\left(t_{0}, t_{1}\right)
$$

Solution of (18) with the initial condition $\varepsilon(0)=\varepsilon_{\mathrm{H}}(0)=\frac{\sigma^{*}}{E}$ taken into account, is

$$
\varepsilon(t)=\frac{\sigma^{*}}{\eta} t+\frac{\sigma^{*}}{E}
$$

Let $t_{0}=0$ be an initial time instant in which the constant stress $\sigma^{*}$ is suddenly imposed and being maintained in time. All absorbed energy is stored by the spring in that time instant due to immediate deformation of the spring. The dashpot initial displacement is zero. So $U(0)=U_{\mathrm{p}}(0)=U_{\mathrm{p}}(t)=\frac{1}{2} E \varepsilon^{2}(0)=\frac{1}{2} E \varepsilon_{\mathrm{H}}{ }^{2}(0)=\frac{1}{2 E} \sigma^{* 2}$. Potential energy remains constant with time lapsing, hence its time derivative is zero, $\dot{U}_{\mathrm{p}}(t)=0$ until the constant load $\sigma^{*}$ persists. Then we can quantify the total energy per volume unit for step wisely loaded Maxwell model as follows:

$$
U(t)=U(0)+\int_{0}^{t} \sigma^{*} \dot{\varepsilon}(a) \mathrm{d} a=\frac{1}{2 E} \sigma^{* 2}+\sigma^{*} \int_{0}^{t} \frac{\sigma^{*}}{\eta} \mathrm{d} a=\sigma^{* 2}\left(\frac{1}{2 E}+\frac{1}{\eta} t\right)
$$


It is apparent from physical point of view that while the constant load persists, the amount of dissipation energy increases linearly with time. This is stated in (20). The dissipated energy rate is $\dot{U}_{\mathrm{d}}(t)=\sigma_{\mathrm{N}}(t) \dot{\varepsilon}_{\mathrm{N}}(t)=\frac{1}{\eta}{\sigma_{\mathrm{N}}}^{2}(t)$, accordingly the dissipation energy can be quantified as follows:

$$
U_{\mathrm{d}}(t)=\int_{0}^{t} \dot{U}_{\mathrm{d}}(a) \mathrm{d} a=\int_{0}^{t} \eta^{-1}{\sigma_{\mathrm{N}}}^{2}(t) \mathrm{d} a=\frac{1}{\eta} \sigma^{* 2} t
$$

We can see easily that for creep test on Maxwell model the equality $U_{d}(t)+U_{\mathrm{p}}(t)=$ $U(t)$ holds.
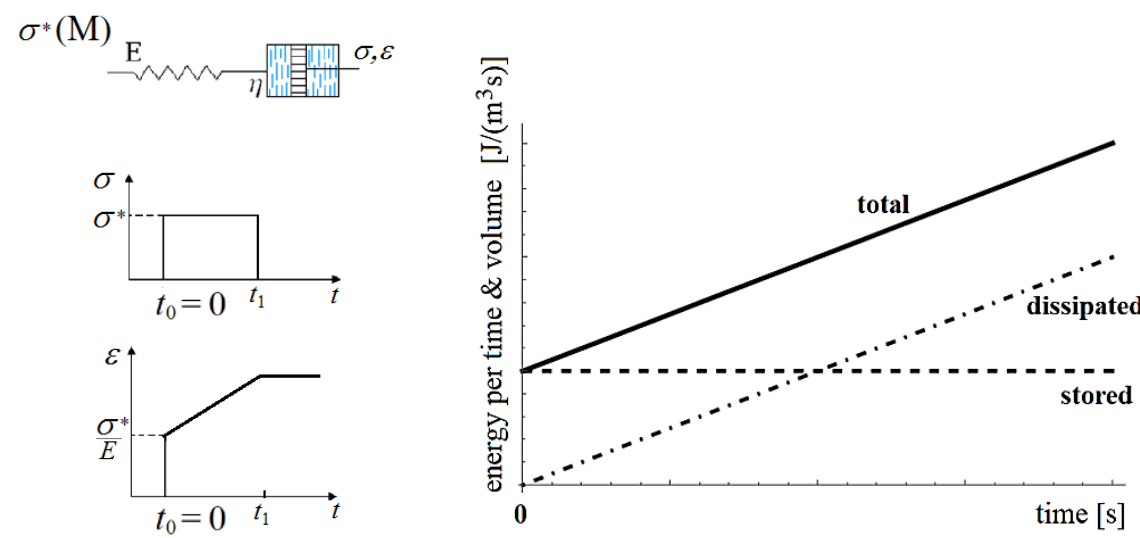

Fig. 4. Maxwell model and its creep test (left): Action $\sigma^{*}$, reaction $\varepsilon(t)$. Total, stored and dissipated energy functions of time within the time interval $\left(t_{0}=0, t_{1}\right\rangle$ (right).

\subsection{Relaxation test on Maxwell model - constant strain imposed}

A constant strain is immediately applied and maintained in time: $\varepsilon(t)=\varepsilon^{*} ; t \in\left(t_{0}=0, t_{1}\right\rangle$. The responding stress function is given as a solution of the differential equation

$$
\frac{\dot{\sigma}(t)}{E}+\frac{\sigma(t)}{\eta}=\dot{\varepsilon}^{*}(t)=0 \text { in }\left(t_{0}=0, t_{1}\right)
$$

As soon as the model is step wisely loaded, the immediate deformation occurs. The initial deformation of $(\mathrm{N})$ is zero. Later on, the deformation of $(\mathrm{N})$ increases together with corresponding decrease of deformation of $(\mathrm{H})$. This process (until persisting deformation load) continues until the elastic element deformation diminishes back to zero, e.g. all deformation of the viscoelastic material is transferred to the viscous element.

The stress function, i.e. the solution of (22) with the initial condition $\sigma(0)=\varepsilon^{*} E$ taken into account is

$$
\sigma(t)=\varepsilon^{*} E \exp \left(-\frac{E}{\eta} t\right)
$$

From (9) we see that $\dot{U}(t)=0$, hence $U(t)=$ const

$$
U(t)=U(0)=U_{\mathrm{p}}(0)=\frac{1}{2} E \varepsilon_{\mathrm{H}}^{2}(0)=\frac{1}{2} E \varepsilon^{* 2}
$$


As physically expected and documented in the following computation, the dissipation energy increases together with the increase of the dashpot deformation; and the potential energy decreases down to zero adequately; as both, potential and dissipation energy in any time instant, are summed up to the total energy $U(t)=U_{\mathrm{p}}(t)+U_{\mathrm{d}}(t)=\frac{1}{2} E \varepsilon^{* 2}$ (constant during all loading time). Since $\sigma_{\mathrm{H}}(t)=\sigma_{\mathrm{N}}(t)=\sigma(t)$, from (23) follows $\varepsilon_{\mathrm{H}}(t)=\frac{\sigma_{\mathrm{H}}(t)}{E}=$ $\frac{\sigma(t)}{E}=\varepsilon^{*} \exp \left(-\frac{E}{\eta} t\right)$ and by substituting for $\varepsilon_{\mathrm{H}}(t)$ to (15) we obtain

$$
\begin{gathered}
U_{\mathrm{p}}(t)=U_{\mathrm{p}}(0)+\int_{0}^{t} \sigma_{\mathrm{H}}(a) \dot{\varepsilon}_{\mathrm{H}}(a) \mathrm{d} a=\frac{1}{2} E \varepsilon^{* 2}+\int_{0}^{t} E \varepsilon^{*}\left(-\frac{E}{\eta}\right) \varepsilon^{*} \exp \left(-\frac{2 E}{\eta} a\right) \mathrm{d} a= \\
\frac{1}{2} E \varepsilon^{* 2}\left(1-\exp \left(-\frac{2 E}{\eta} t\right)\right)
\end{gathered}
$$

As $\dot{\varepsilon}_{\mathrm{N}}^{2}(t)=\frac{\sigma_{\mathrm{N}}{ }^{2}(t)}{\eta^{2}}$ and $\sigma_{\mathrm{N}}(t)=\sigma(t)$, we have next the dissipation energy rate and dissipated energy itself for $(\mathrm{M})$ subjected to deformation load constant in time:

$$
\begin{gathered}
\dot{U}_{\mathrm{d}}(t)=\eta \dot{\varepsilon}_{\mathrm{N}}^{2}(t)=\frac{\varepsilon^{* 2} E^{2}}{\eta} \exp \left(-\frac{2 E}{\eta} t\right) \\
U_{\mathrm{d}}(t)=\int_{0}^{t} \sigma_{\mathrm{N}}(a) \dot{\varepsilon}_{\mathrm{N}}(a) \mathrm{d} a=\int_{0}^{t} \frac{E^{2}}{\eta} \varepsilon^{* 2} \exp \left(-\frac{2 E}{\eta} a\right) \mathrm{d} a=\frac{\varepsilon^{* 2} E}{2} \exp \left(-\frac{2 E}{\eta} t\right)
\end{gathered}
$$

$\varepsilon^{*}(\mathrm{M})$
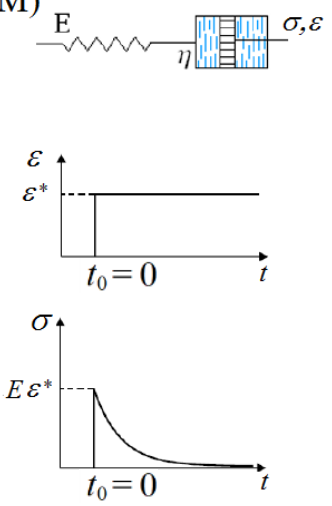

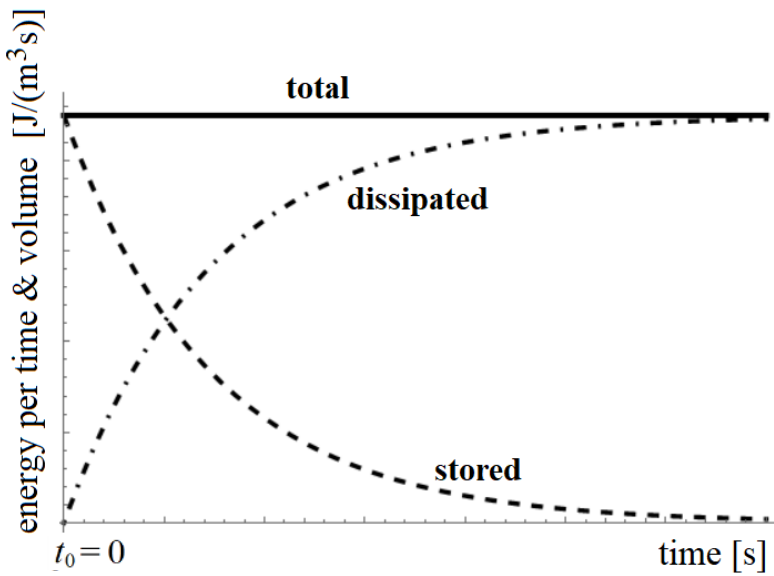

Fig. 5. Maxwell model and its relaxation test (left): Action $\varepsilon^{*}$, reaction $\sigma(t)$. Total, stored and dissipated energy - functions of time (right).

We can easy see, that both stored (25) and dissipated (26) energy are summed up to the total (24) deformation energy.

Finally it is worth mentioning that forms (9) - (14) are general for Maxwell model, accordingly any loading function of time can be taken as the action - either $\sigma(t)$ or $\varepsilon(t)$ yielding the total, stored and dissipation energy functions matching with the mechanical response, reaction of the Maxwell model.

\section{Conclusion}

In this work the recent investigation of author is presented dealing with thermodynamic aspects of the viscoelastic bodies represented by their models. First a general consideration 
concerning the thermodynamics in rheology is provided together with the supporting formulas derivation. Then the Maxwell model is studied from the thermodynamic point of view. The resulting forms and graphical performance document the validity of the previous theoretical consideration.

Nowadays, the prediction of behaviour of materials under various types of load is of a great significance in material science. The theoretical evaluation of corresponding energy can help efficiently with the energy consumption prediction of new soft materials.

This work is supported by grant VEGA 1/0456/17 and APVV-18-0052.

\section{References}

1. M. Minárová: Slov. J. of Civil Eng.ineering, 22(4), pp. 19-24 (2014)

2. M. Minárová: Rheology. Viscoelastic and viscoelastoplastic modelling. Habilitation thesis, Bratislava (2018)

3. M. Minárová and J. Sumec: Advanced and Trends in Engineering Science and Technologies, pp. 207-212 (2016)

4. R.M. Christensen: Theory of Viscoelasticity, An Introduction, (in Russian), Mir, Moscow (1974)

5. P. Krejčí, Hysteresis, Convexity and Dissipation in Hyperbolic Equations, Gakuto Intern. Ser. Math. Sci. Appl., 8 (1996).

6. J. Kopfová, M. Minárová and J. Sumec: Proceedings of Equadiff conference 2017, SPECTRUM STU, pp. 173-180 (2018)

7. J.N. Rabotnov: Creep Problems in Structural Members, North-Holland, Amsterdam (1969)

8. N.W. Tschoegl: The Phenomenological Theory of Linear Viscoelastic Behavior, Springer-Verlag New York, (1989) 